\title{
ASSESSMENT OF TRAFFIC DISRUPTION CAUSED BY URBAN FLOODING
}

\author{
DONG HO KANG, KYUNG SU CHOO \& BYUNG SIK KIM \\ Department of Urban and Environmental Disaster Prevention Engineering, \\ Kangwon National University, South Korea
}

\begin{abstract}
Recently, local heavy rainfall and typhoons have increased the level of human and property damage in urban environments. Moreover, inundation due to urban flooding has led to severe traffic network disruption and accidents. Rainfall is considered one of the key factors influencing vehicle speed. Thus, in this study, we developed a rainfall $(\mathrm{mm})$-inundation depth $(\mathrm{cm})$ curve formula to predict changes in vehicle speed under urban flooding. To produce the inundation depth data, rainfall over 10-200 mm was increased by $10 \mathrm{~mm}$, and the respective runoff data were calculated using S-RAT (Spatial Runoff Assessment Tool), a distributed runoff model. Next, the simulated runoff data was inputted to the flood inundation model, Flo-2D. With the use of such assessed formula and previous research on the rainfall $(\mathrm{mm})$-vehicle speed $(\mathrm{km} / \mathrm{h})$ relation, the rainfall $(\mathrm{mm})$-inundation depth $(\mathrm{cm})$-vehicle speed $(\mathrm{km} / \mathrm{h})$ curve formula was created. The pilot areas were Seoul metropolitan in South Korea, which was divided into $1 \times 1 \mathrm{~km}$ grids. Finally we could analyse the impact of urban flooding on traffic disruption. According to the analysis, the flooding area during the time of the actual rainfall was found to have poor traffic, but there are some areas where the speed was not reduced. The results from the flood depth-vehicle speed curve could be derived by reflecting the hydrological characteristics of each grid, but the curves for the rainfall-vehicle speed formula did not reflect the characteristics, resulting in most grids having similar patterns. Through this process, a traffic reduction rate map was developed and the speed reduction by section was suggested.

Keywords: urban flood, rainfall (mm)-flood depth $(\mathrm{m})$ curve, rainfall ( $\mathrm{mm})$-flood depth (m)-vehicle speed $(\mathrm{km} / \mathrm{h})$ curve, traffic disruption, impact assessment.
\end{abstract}

\section{INTRODUCTION}

Recent advances in technology have made it possible to provide rapid and accurate information to the public by obtaining more reliable and widely spread rainfall data and processing and analyzing collected rainfall data through various equipment. Furthermore, studies are being actively conducted to analyze places where a lot of vehicles are related and to recommend destinations through different routes through real-time data. However, studies on the traffic situation prediction using rainfall data and studies on the speed of roads in flooded urban roads are also lacking. The purpose of this study is to analyze the relationship between flood depth-vehicle speed rather than rainfall-vehicle speed. Later, a risk map can be created to help the route bypass if a rain event occurs and the road is flooded. In this study, the rainfall-flood depth-vehicle speed curve was calculated using the rainfall-flood depth curve and the flood depth-vehicle speed curve. In 2011, the rainfall was inspected for flooding in the Sadang-dong area of Seoul.

Jeong et al. [1] analyzed the traffic characteristics according to precipitation using RWIS (Road Weather Information System) data and detector data, and suggested that speed and traffic volume decreased when rainfall occurred. Speed estimation curves according to rainfall intensity were presented, and the criteria for low and medium precipitation were $0.4 \mathrm{~mm} / 5 \mathrm{~min}$, and the criteria for moderate and strong precipitation were $0.8 \mathrm{~mm} / 5 \mathrm{~min}$ when rain levels were classified using the reduction in speed according to rainfall. Lam et al. [2] proposed a speed-density relation under various rainfall conditions considering vehicle speed, speed according to traffic volume, and traffic volume that can be accommodated by 
road, and suggested a function formula of vehicle speed and traffic volume according to rainfall intensity. In Hong Kong and other Asian cities, it is suggested that rainfall intensity should be considered in the design, operation, and evaluation of road facilities because the average annual rainfall intensity is relatively high. Mashros et al. [3] estimated the effect of rainfall on the moving speed and the extent of the speed decrease using three months of data. Thus, the rainfall intensity is divided into four stages and a graph shows that the moving speed decreases in percentiles of 15,50 , and 85 as the rainfall intensity increases. Kim and Oh [4] used the traffic volume data of the general national road and the rain data of the Korea Meteorological Administration to conduct a study on the daily mean traffic variation by rainfall intensity (weekly, weekend) and suggested that the more the rainfall, the more the average daily traffic varies by up to $5.48 \%$ in all days.

\section{THEORETICAL BACKGROUND}

\subsection{Rainfall-flood depth curve}

The rainfall-flood depth curve is a curve using the flood depth, which is the result of the rainfall data provided by the Korea Meteorological Agency and the modeling results. In order to calculate the depth of flooding, first a rainfall runoff model was used with the rainfall of $10-200 \mathrm{~mm}$ as the Huff quartile method. The estimated runoff is applied to the flood overflow model and the rainfall-flood depth curve is created using the flood depth data. See Lee et al. [5] for further analysis of the rainfall-depth curve.

\subsection{Rainfall-flood depth-vehicle speed curve}

In this study, the flood depth-vehicle speed curve was obtained by using the data from a previous analysis between the flood depth and the speed of the vehicle. The estimation function data presented in Pregnolato et al. [6] were used to calculate the flood depth-vehicle speed curve. This lead to the linear regression equation

$$
\mathrm{y}=85 \times e^{-9 x}\left(R^{2}=0.87\right) .
$$

\section{APPLICATION TO THE STUDY BASIN}

\subsection{Study basin}

To carry out this study, Seoul City, the most urbanized city in Korea, was selected as the target area. The city area was divided into $1 \mathrm{~km} \times 1 \mathrm{~km}$ grids along the shrine, Bangbae, and Seocho-dong, which were affected by a heavy rain of about $300 \mathrm{~mm}$ from 1:00 am to 11:00 pm on 27 July 2011. Fig. 1 shows the area of Sadang-dong, which is divided into grids. Fig. 2 shows the grid number, road network, and speed limit for each grid in Sadang-dong. In this study, we analyzed grids 23 and 26 where actual damage had occurred in the past.

\subsection{Rainfall-flood depth curve calculation results}

In order to calculate the rainfall-flood depth curve, the area of Sadang-dong was divided into $1 \mathrm{~km} \times 1 \mathrm{~km}$ grid and numbers were assigned to each grid square. The vehicle speed was set based on the road speed limit of each grid square. Most of them included roads in downtown Seoul, indicating that there were many $60 \mathrm{~km}$ speed limit roads. Figure 3 shows the calculation of the rainfall-depth curve in grid 23 and 26 where the damage occurred. 

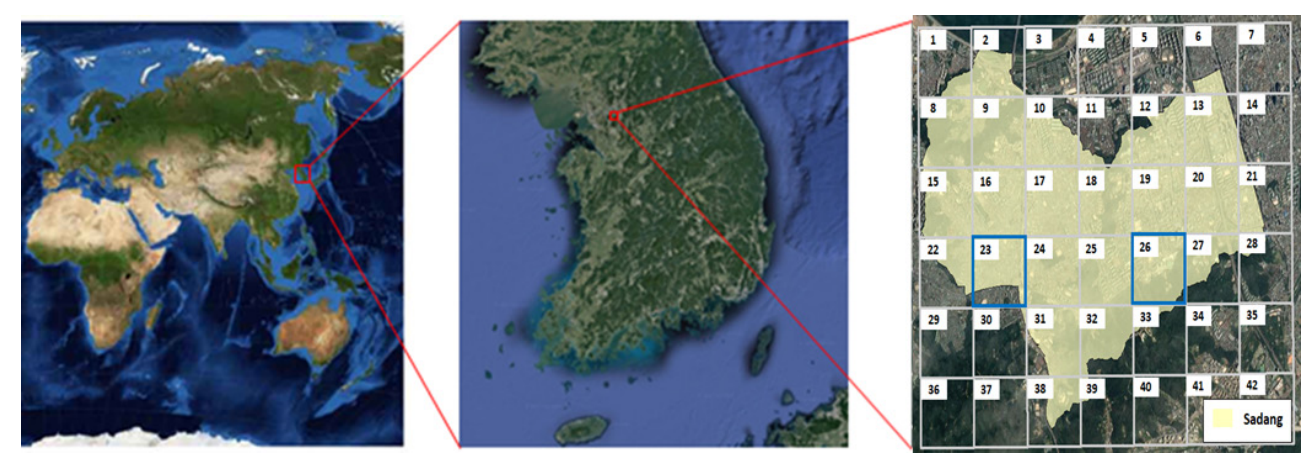

Figure 1: Study basin.

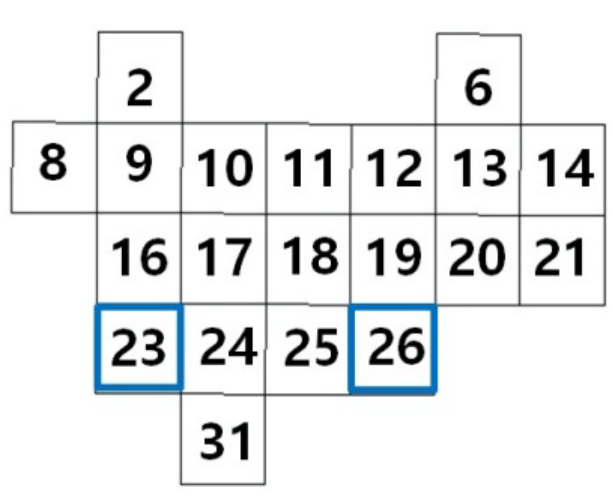

(a)

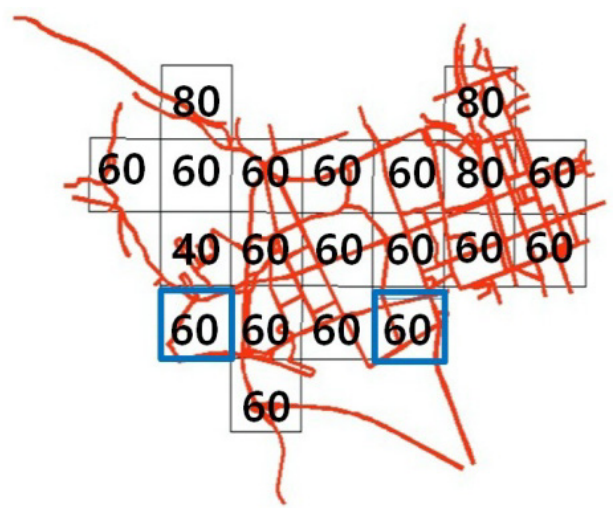

(b)

Figure 2: $\quad$ Sadang-dong grid number and speed limit for each grid. (a) Grid number; and (b) Speed limit for each grid.

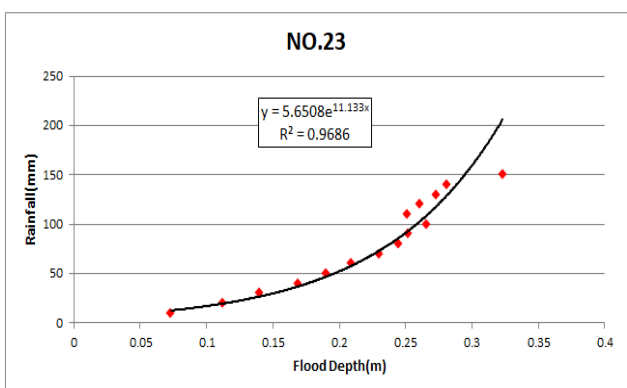

(a)

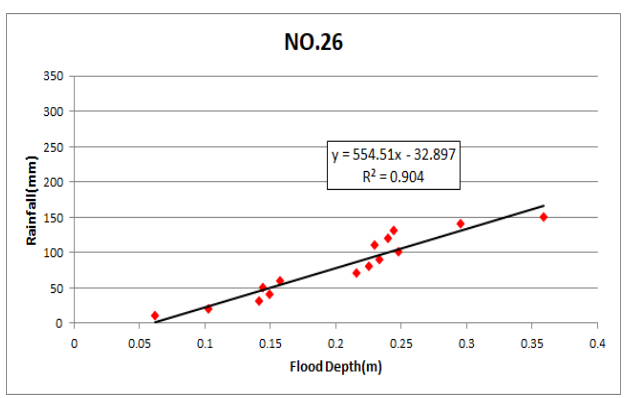

(b)

Figure 3: Result of rainfall-depth curve. 


\subsection{Results of estimation of rainfall-flood depth-vehicle speed curve}

On 27 July 2011, there was a traffic restriction due to flooding at 6:30 am at the Sadang Station included in the 23rd grid, and traffic resumed at 10:00 am. As a result of the rainfallflood depth-vehicle velocity curve calculation, it was predicted that a vehicle could not travel at the time when the actual damage occurred, and it can be confirmed that the traffic resumes after damage time. Figure 4 shows the rainfall-flood depth-vehicle curve result.
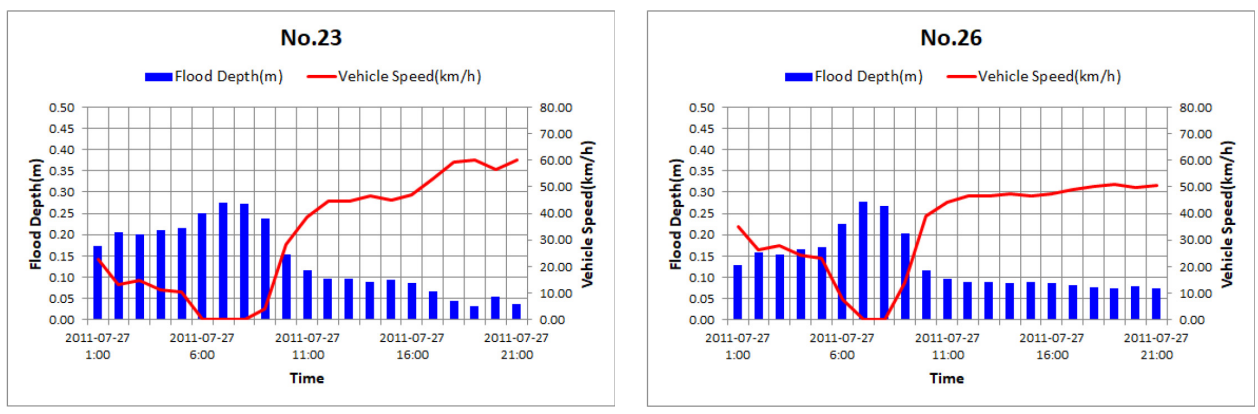

Figure 4: Rainfall-flood depth-vehicle speed curve.

\subsection{Traffic disruption impact assessment by grid}

The rate of reduction of vehicle speed by grid was calculated using the calculated rainfallflood depth-vehicle speed curve. Traffic disruption impact assessment was carried out by dividing the rate of reduction of vehicle speed into four levels of very low, low, medium and high. The criteria for dividing into four grades were calculated as the rate of decrease according to the speed limit for each grid. Very low has a reduction rate of $0-25 \%$, low $25-$ $50 \%$, medium $50-75 \%$, and high $75-100 \%$. Fig. 5 is a picture of the traffic disruption rating of the time when the vehicle was controlled by waterlogging at 7:00 am on 27 July 2011 and the traffic disruption rating at 1:00 pm when vehicle control was lifted. It can be seen that the

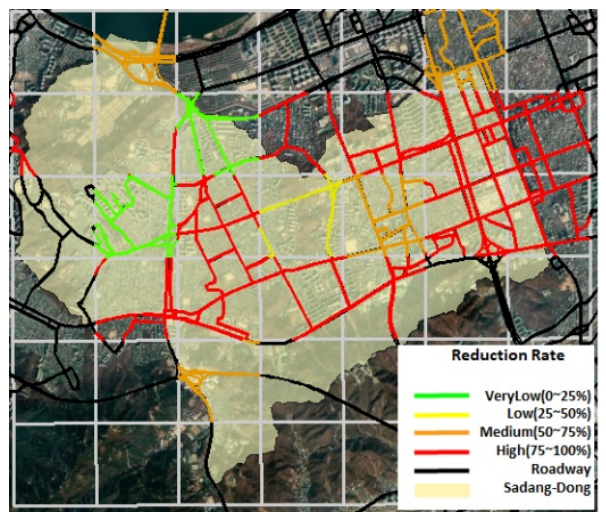

(a)

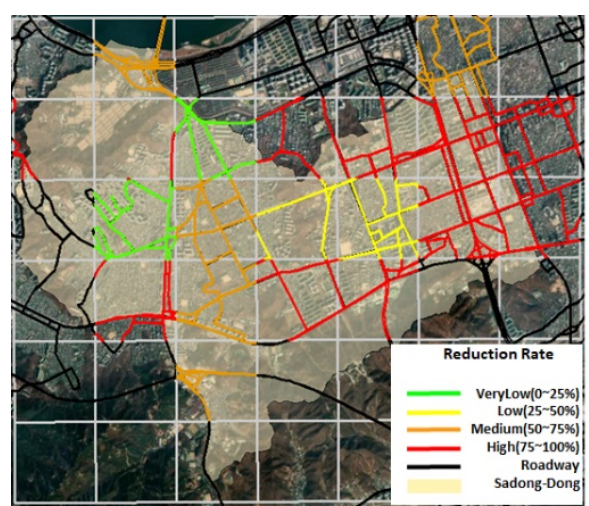

(b)

Figure 5: Analysis of impact assessment. (a) 7:00 am on 27 July 2011 traffic disruption rating; and (b) 9:00 am on 27 July 2011 traffic disruption rating. 
vehicle reduction rate is high in many grids at the time of the volume control, and that there is a grid square having smooth traffic due to the low vehicle reduction rate. This can allow bypassing roads with high rates of reduction so that drivers can reach their destination.

\section{CONCLUSION}

In this study, the rainfall-flood depth-vehicle speed curve was calculated using the rainfallflood depth curve and the flood depth-vehicle speed curve. This curve is generated by time unit and the result can be confirmed. For the verification of the calculated curves, the test was conducted using the 27 July 2011 event, at which actual damage occurred in the past.

The results of the verification confirmed that traffic was not possible in the rainfall-flood depth-vehicle speed curve from 6:00 am when actual vehicle traffic was controlled until 10:00 am when traffic was resumed. In this study, only two grid squares were used for model verification, but if there are additional articles, news, and damage pictures that actually occurred, it is necessary to further verify the model. Rainfall-flood depth-vehicle speed curves still need a lot of complementary work. It is expected to increase the practical utilization if the individual's psychology and behavioral patterns such as the driver's understanding the characteristics of the rain and thus further develop the relational expression.

Using a proven curve, the traffic disturbance class by time zone was determined to be four stages from 1:00 am to 11:00 pm on 27 July 2011, and there was a smooth traffic grid square during the time when vehicle control was carried out due to flooding as a result of the calculation, which can be utilized in various ways in the future.

If a public servant uses this study, it is expected that the budget for road maintenance can be organized according to the proportion of road connectivity (where most pass by when vehicles go elsewhere) by upgrading each road characteristics and drain pipe network.

In this study, the depth of flooding can be calculated and the vehicle speed can be analyzed according to the predicted rainfall. It is still in the early stages of development, but, it is believed that can continue if the recommended route can be taken into account by considering the speed of the vehicle and the minimum cost, and if actual flooding can be used to identify its effect.

\section{ACKNOWLEDGEMENTS}

This work was funded by the Korea Meteorological Administration Research and Development Program under grant KMI[2018-03010]. This work was supported by Korea Environment Industry and Technology Institute (KEITI) through Advanced Water Management Research Program, funded by Korea Ministry of Environment (MOE) (83091). This paper was financially supported by Ministry of the Interior and Safety as "Human Resource development Project in Disaster Management".

\section{REFERENCES}

[1] Jeong, E., Oh, C. \& Hong, S., Prediction of speed by rain intensity using road weather information system and vehicle detection system data. Intelligent Transport Systems, 12(4), pp. 44-55, 2013.

[2] Lam, W.H.K., Tam, M.L., Cao, X. \& Li, X., Modeling the effects of rainfall intensity on traffic speed, flow, and density relationships for urban roads. Journal of Transportation Engineering, 139(7), pp. 758-770, 2013.

[3] Mashros, N., Ben-Edigbe, J., Alhassan, H.M. \& Hassan, S.A., Investigating the impact of rainfall on travel speed. Journal Teknologi, 71(3), pp. 33-38, 2014. 
[4] Kim, T.W. \& Oh, J.S., Analysis of provincial road in national highway average speed variation according to rainfall intensity. Journal of the Korea Contents Association, 15(4), pp. 510-518, 2015.

[5] Lee, S.H., Kang, D.H. \& Kim, B.S., A study on the method of calculating the threshold rainfall for rainfall impact forecasting. Journal of the Korean Society of Hazard Mitigation, 18(7), pp. 93-102, 2018.

[6] Pregnolato, M., Ford, A., Wilkinson, S.M. \& Dawson, R., The impact of flooding on road transport: A depth-disruption function. Transportation Research Part D: Transport and Environment, 55, pp. 67-81, 2017. 